\title{
In vitro and in vivo effect of 3-Para-fluorobenzoyl-propionic acid on rat liver mitochondrial permeability transition pore opening and lipid peroxidation
}

\author{
*Adeola O. Olowofolahan, Omosola L. Bolarin and Olufunso O. Olorunsogo
}

Laboratory for Membrane Biochemistry Research and Biotechnology, Department of Biochemistry, University of Ibadan, Nigeria.

Received $17^{\text {th }}$ February, 2020, Accepted 15 ${ }^{\text {th }}$ May, 2020

DOI: 10.2478/ast-2020-0005

*Corresponding author

Adeola O.Olowofolahan E-mail:mradeola@yahoo.com

Tel: +234- 7030179598

\begin{abstract}
The opening of mitochondrial permeability transition (mPT) pore is a well recognized important event in the execution of mitochondrial-mediated apoptosis. Some bioactive compounds induce apoptosis in tumour cells via the induction of mPT pore opening. This study therefore investigated the effect of 3-Para-fluorobenzoyl-propionic acid (3PFBPA), a metabolite of haloperidol on mPT pore, mitochondrial ATPase activity (mATPase), mitochondrial lipid peroxidation (mLPO) and cytochrome c release (CCR). Thirty-two male Wistar rats, were acclimatized for 14 days in clean cages. After 30 days of treatment, they were sacrificed and the liver mitochondria isolated using differential centrifugation. The mPT pore, mATPase, mLPO and CCR were determined by standard methods using a spectrophotometer. The mPT pore opening was induced by 3PFBPA by 1.4, 3.6, 5.6, 6.6 and 7.4 folds, when compared with the control. Also, there was release of cytochrome c and enhancement of mATPase activity by 3PFBPA. The results also show that 3PFBPA reduced lipid peroxidation. However, oral administration of 3PFBPA at 50,100 and $200 \mathrm{mg} / \mathrm{kg}$ did not have any effect on $\mathrm{mPT}$ pore opening and mATPase activity when compared with the control but there was inhibition of mLPO. These findings suggested the pharmacological potential of 3PFBPA against the pathological processes related to insufficient apoptosis (based on the in vitro data) and oxidative stress due to its anti-lipidperoxidative effect.
\end{abstract}

Keywords: 3-(Para-fluorobenzoyl)-propionic acid, mitochondrial permeability transition pore, apoptosis. 


\subsection{Introduction}

Apoptosis is one of the defence mechanisms by which deleterious and mutated cells are removed from an organism to maintain proper growth and development, differentiation, metamorphosis and embryogenesis. (Mcllwain et al., 2013). Impairment of apoptotic mechanism is now being implicated in many diseased conditions including neurodegenerative diseases, cancer, etc (Reed, 2004). The mitochondria are now well recognized in the execution of mitochondrial-mediated apoptosis (MdSoriful et al., 2017). The mitochondrion is composed of inner and outer membrane. The permeabilization of mitochondrial outer membrane is a major event in the induction of mitochondrial-mediated apoptosis (Wang, 2012; Olowofolahan et al., 2019). The induction of mitochondrial membrane permeability transition (mPT) pore can cause mitochondrial swelling, release of cytochrome $\mathrm{c}$ and formation of apoptosome which would ultimately lead to cell death. This involves movement of some apoptogenic proteins from mitochondrial intermembrane space into the cytosol and consequently resulting in cell death (Kallenberger et al., 2014). Studies have shown that certain bioactive agents elicit their chemo-protective effect against tumour via induction of mitochondrial permeability transition (mPT) pore opening. Examples include polyphenols such as resveratrol, epigallocatechin gallate (EGCG) and vanilloids (including capsaicin and curcumin) (Martins, 2006).

3-(para-fluorobenzoyl)-propionic acid (3PFBPA) shown in figure 1 is one of the metabolites of haloperidol which is an anti-psychotic drug. The 3PFBPA is an inhibitor of mitogen activated protein kinase (MAPK) signaling (HyeonSoo et al., 2006), which is implicated in the development of uterine fibroid and cellular proliferation (Huang et al., 2012). It is not known whether 3PFBPA which is an inhibitor of MAPK would cause induction of $\mathrm{mPT}$ pore opening and thus be relevant in situations that require upregulation of mitochondrial cell death as the pore now serves as a pharmacological target for drug development (Jiang et al., 2013). This study was therefore designed to determine the effect of 3PFBPA on mitochondrial-mediated apoptosis via the induction of $\mathrm{MPT}$ pore opening.

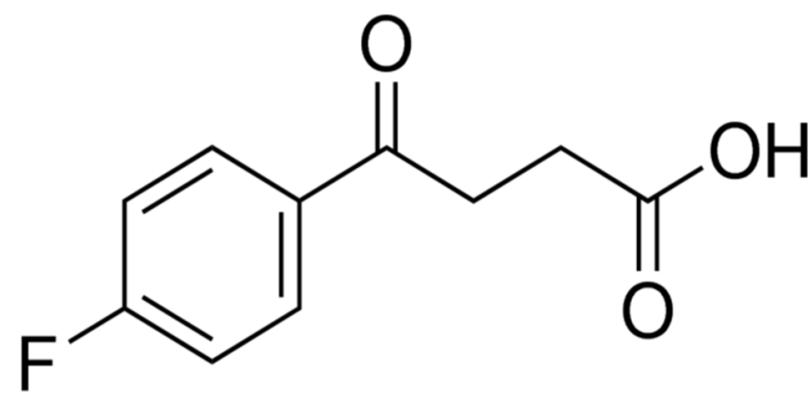

Figure 1: 3-para-fluorobenzoyl-propionic acid (Bowen et al., 1990)

\subsection{Experimental}

\section{Chemicals and Reagents}

The 3-(para-fluorobenzoyl)-propionic acid and all other reagents were purchased from Sigma-Aldrich Chemical Co. (St. Louis, MO, USA) and were of the highest purity grade available.

\section{Experimental Animals}

Male Wistar strain albino rats weighing between 80-90g were purchased from the Preclinical Animal House of the College of medicine, University of Ibadan, Ibadan, Nigeria. All the animals were allowed to acclimatize for a period of two weeks in the Animal House of the Department of Biochemistry, University of Ibadan. The animals were placed under a $12 \mathrm{hr}$ light/dark cycle and fed commercial pelletized rat chow and water ad libitum throughout the experimental period. The work was approved by the Departmental Ethical Review Committee and was conducted according to the guidelines of National Institute of Health (NIH publication 85-23, 1985) for laboratory animal care and use.

\section{Experimental design}

Thirty two male Wistar strain albino rats were randomly assigned into 4 equal groups, after which they were treated with 3PFBPA for 30 days. The stock solution of 3PFBPA was prepared by dissolving $0.75 \mathrm{~g}$ of 3PFBPA in $20 \mathrm{ml}$ of ( $5 \%$ DMSO) corn oil. Based on the weight of the animals, 50, 100 and $200 \mathrm{mg} / \mathrm{kg}$ dosage of 3PFBPA was administered to the treatment groups.

Dose Regimen:

Grp 1: Control (Corn oil containing 5\% DMSO)

Grp 2: $50 \mathrm{mg} / \mathrm{kg}$ 3PFBPA

Grp 3: $100 \mathrm{mg} / \mathrm{kg}$ 3PFBPA

Grp 4: $200 \mathrm{mg} / \mathrm{kg}$ 3PFBPA

One day after the final exposure, the animals were sacrificed.

\section{Rat liver mitochondria isolation}

Rat liver mitochondria were isolated as described and modified by Olorunsogo et al. (1979). The animals were sacrificed by cervical dislocation and the livers excised and trimmed to wash excess tissue. The livers were then weighed, washed with homogenizing buffer (210mM Mannitol, 70mM Sucrose, 5mM HEPES-KOH, pH 7.4 and $1 \mathrm{mM}$ EGTA), and homogenized as a $10 \%$ suspension in ice -cold buffer using a Porter Elvehjem glass homogenizer. The resulting homogenate was centrifuged in an MSE refrigerated centrifuge at $2300 \mathrm{rpm}$ for 5 minutes to remove the nuclear debris. This was done twice and the supernatant obtained was centrifuged at $13,000 \mathrm{rpm}$ for 10 minutes to obtain the mitochondrial pellet. The supernatant was discarded while the pellet was washed twice with the washing buffer $(210 \mathrm{mM}$ Mannitol, 70mM sucrose, 5mM HEPES-KOH, pH 7.4, 0.5\% BSA) at $12,000 \mathrm{rpm}$ for 10 minutes. The mitochondria obtained were immediately resuspended in an appropriate volume of MSH buffer (210mM Mannitol,70mM sucrose, 5mM HEPES-KOH, pH 7.4), and immediately dispensed into eppendorf tubes and kept on ice. 


\section{Determination of mitochondrial protein}

Mitochondrial protein concentration was determined according to the method of Lowry et al (1951), using bovine serum albumin as standard.

\section{Assessments of FoF1 ATPase Activity}

$\mathrm{F}_{0} \mathrm{~F}_{1}$ Adenosine triphosphatase was determined by a modification of the method of Olorunsogo and Malomo (1985). Each reaction mixture contained $65 \mathrm{mM}$ Tris- $\mathrm{HCl}$ buffer $\mathrm{pH} 7.4,0.5 \mathrm{mM} \mathrm{KCl} 1 \mathrm{mM}$ ATP and $25 \mathrm{mM}$ sucrose. The reaction mixture was made up to a total volume of $2 \mathrm{ml}$ with distilled water. The reaction was started by the addition of mitochondrial suspension and was allowed to proceed for 30 minutes at $27^{\circ} \mathrm{C}$. The reaction was stopped by the addition of $1 \mathrm{ml}$ of a $10 \%$ solution of sodium dodecyl sulphate. The zero time tube was prepared by adding the solution of ATP to the reaction vessel following the addition of sodium dodecyl sulphate. 2,4 Dinitrophenol (2,4 DNP) was used as a standard uncoupling agent.

\section{Estimation of Inorganic phosphate released}

The concentration of inorganic phosphate released following the hydrolysis of ATP was determined according to the method described by Bassir (1965) and as modified by Olorunsogo et al. (1979). Aliquot of each solution $(300 \mu \mathrm{l})$ was dispensed into fresh test tubes, followed by the addition of $300 \mu \mathrm{l}$ of distilled water. To each of the test tube, 1 $\mathrm{ml}$ of $5 \%$ ammonium molybdate and $1 \mathrm{ml}$ of $9 \%$ freshly prepared solution of ascorbic acid were added. The tube was well mixed and allowed to stand for 20 minutes. The absorbance was read at $680 \mathrm{~nm}$. A water blank was used to set the spectrophotometer at zero.

\section{Assay of Cytochrome C release}

The quantitative determination of cytochrome $\mathrm{C}$ released from isolated mitochondria was performed by measuring the Soret $(\gamma)$ peak for cytochrome $c$ at $414 \mathrm{~nm}\left(\varepsilon=100 \mathrm{mM}^{-1} \mathrm{~cm}^{-1}\right)$, according to method of Appaix et al. (2000). Mitochondria (1mg protein/ml) were preincubated in the presence of $0.8 \mu \mathrm{M}$ rotenone in a medium containing $210 \mathrm{mM}$ mannitol, $70 \mathrm{mM}$ sucrose and $5 \mathrm{mM}$ HEPES-KOH $(\mathrm{pH} 7.4)$ for 30 minutes at $27^{\circ} \mathrm{C}$ in the presence of different concentrations of the fractions, using $24 \mathrm{mM}$ calcium as the standard (TA). After the incubation, the mixture was centrifuged at 15,000 rpm for 10 minutes. The optical density of the supernatant was measured at $414 \mathrm{~nm}$ which is the soret $(\gamma)$ peak for cytochrome c.

\section{Assessment of malondialdehyde}

Lipid peroxidation was determined by measuring the formation of thiobarbituric acid reactive substances (TBARS) present in the test sample according to the method of Varshney and Kale (1990). Under acidic conditions, malondialdehyde (MDA) produced from the peroxidation of fatty acids reacts with the chromogenic reagent 2thiobarbituric acid to yield a pink coloured complex with maximum absorbance at $532 \mathrm{~nm}$. An aliquot of $0.4 \mathrm{ml}$ of the test sample was mixed with $1.6 \mathrm{ml}$ of Tris-KCl buffer to which $0.5 \mathrm{ml}$ of $30 \%$ TCA was added. Then $0.5 \mathrm{ml}$ of $0.75 \%$ TBA was added and placed in a water bath for 45 minutes at $80^{\circ} \mathrm{C}$. This was then cooled in ice to room temperature and centrifuged at $3000 \mathrm{rpm}$ for $10 \mathrm{~min}$. The clear supernatant was collected and absorbance measured against a reference blank of distilled water at $532 \mathrm{~nm}$.

\section{Statistical Analysis}

Values were considered statistically significant at a 95\% confidence level when the value was $P \leq 0.05$. Comparison between the control and the treated groups was performed using the Turkey's test. Multiple comparisons were performed using the Graphpad Prism 6 software. Comparison of the variables were made using the ANOVA

\subsection{Results}

As shown by figure 2, there was no change in the absorbance over a period of 12 minutes. However, upon addition of exogenous calcium (Triggering Agent), there was large amplitude mPT pore opening which was significantly reversed by spermine, a standard inhibitor.

As shown in figure 3, there was concentration-dependent induction of pore opening by $1.4,3.6,5.6,6.6$ and 7.4 folds at the various concentrations used respectively, when compared with the control.

The effect of 3PFBPA was depicted in figure 4. Also, there was increase in the cytochrome $c$ released in a concentration-dependent manner. Similar pattern of result was recorded on its effect on mitochondrial ATPase activity. As revealed in figure 5, there was increase in the mitochondrial ATPase activity by varying concentrations of 3PFBPA. Its effect on lipid peroxidation as depicted in figure 6 showed concentration-dependent inhibition of lipid peroxidation. In converse, oral administration of 3PFBPA did not cause any induction of mPT pore opening and there was no significant enhancement of specific activity. Although, there was inhibition of lipid peroxidation as shown in figures 7,8 and 9.

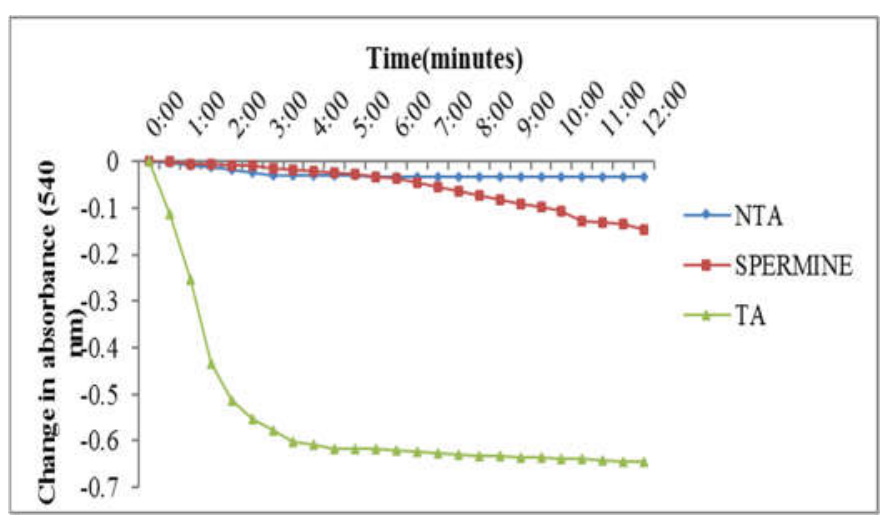

Figure 2: Calcium-induced mitochondrial membrane permeability transition pore opening in normal rat liver mitochondria and its reversal by spermine.

NTA: No triggering agent (without calcium)

TA: Triggering agent (calcium)

Spermine: Standard inhibitor 


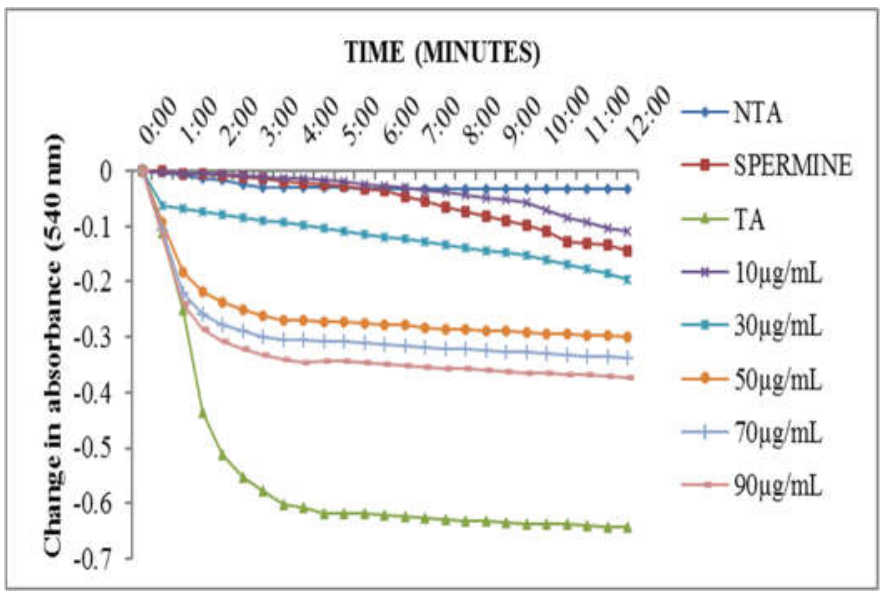

Figure 3: Varying concentrations of 3PFBPA induced rat liver mitochondrial permeability transition pore opening.

3PFBPA: 3-para-fluorobenzoyl-propionic acid

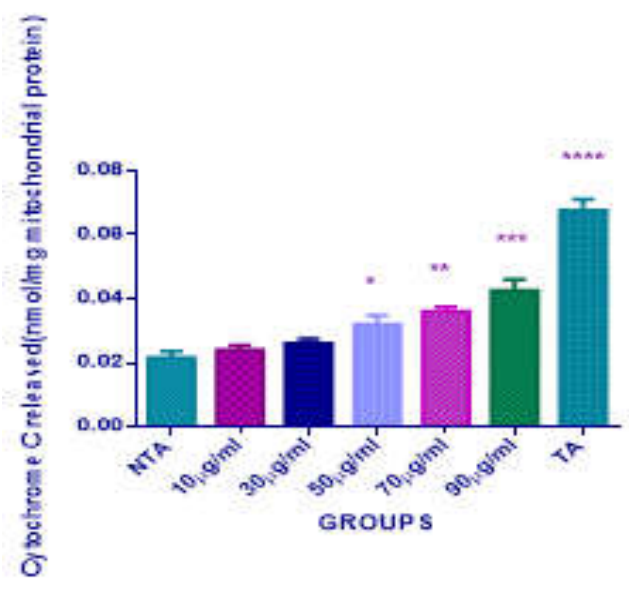

Figure 4: Varying concentrations of 3PFBPA caused the release of cytochrome c.

3PFBPA: 3-para-fluorobenzoyl-propionic acid

* Each value is statistically significant at $\mathbf{p}<0.05$, compared with control using the one-way ANOVA

** Each value is statistically significant at $\mathbf{p}<0.01$, compared with control using the one-way ANOVA

*** Each value is statistically significant at $\mathbf{p}<0.001$, compared with control using the one-way ANOVA

**** Each value is statistically significant at $\mathbf{p}<0.0001$, compared with control using the one-way ANOVA

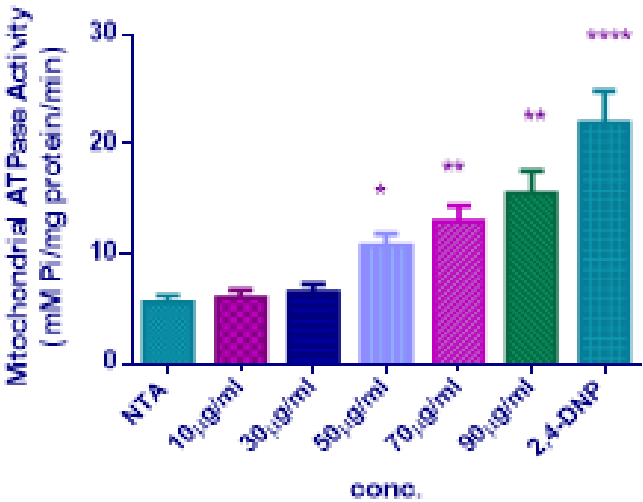

Figure 5: Varying concentrations of 3PFBPA enhanced mitochondrial ATPase activity.

3PFBPA: 3-para-fluorobenzoyl-propionic acid

* Each value is statistically significant at $\mathbf{p}<0.05$, compared with control using the one-way ANOVA

** Each value is statistically significant at $\mathbf{p}<0.01$, compared with control using the one-way ANOVA

$* * * *$ Each value is statistically significant at $\mathbf{p}<0.0001$, compared with control using the one-way ANOVA

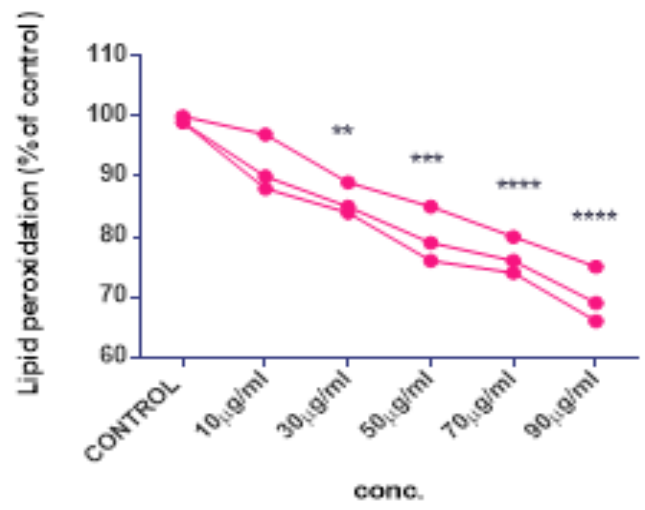

Figure 6: Varying concentrations of 3PFBPA inhibited mitochondrial lipid peroxidation.

3PFBPA: 3-para-fluorobenzoyl-propionic acid

${ }^{* *}$ Each value is statistically significant at $\mathbf{p}<0.01$, compared with control using the one-way ANOVA

*** Each value is statistically significant at $\mathbf{p}<0.001$, compared with control using the one-way ANOVA

**** Each value is statistically significant at $\mathbf{p}<0.0001$, compared with control using the one-way ANOVA 


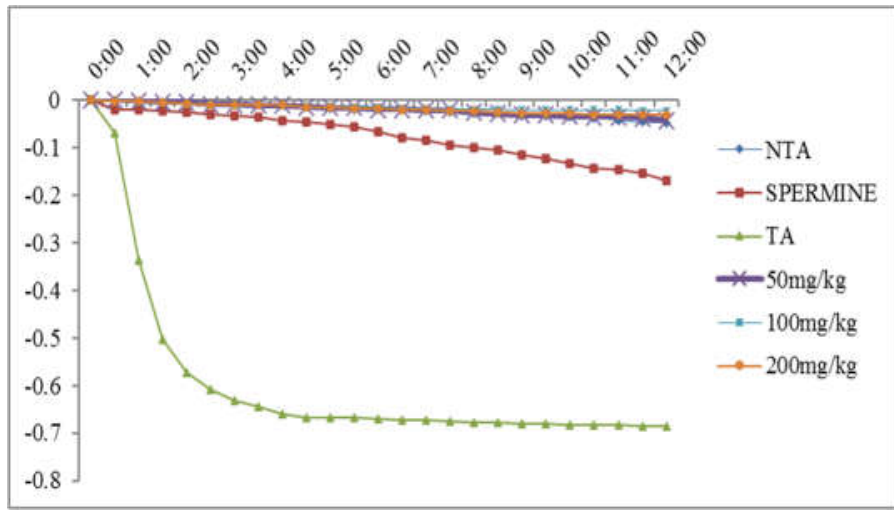

Figure 7: Oral administration of 3PFBPA showed no inductive effect on mitochondrial permeability transition pore.

3PFBPA: 3-para-fluorobenzoyl-propionic acid

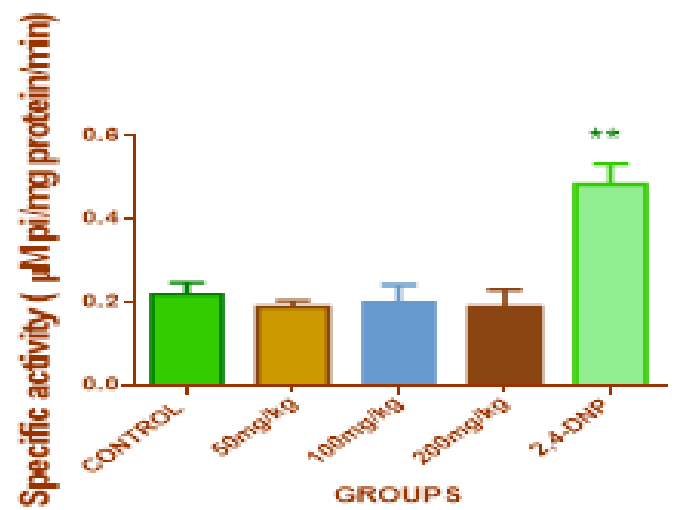

Figure 8: Oral administration of 3PFBPA showed no significant effect on mitochondrial ATPase activity .

3PFBPA: 3-para-fluorobenzoyl-propionic acid

** Each value is statistically significant at $\mathbf{p}<0.01$, compared with control using the one-way ANOVA

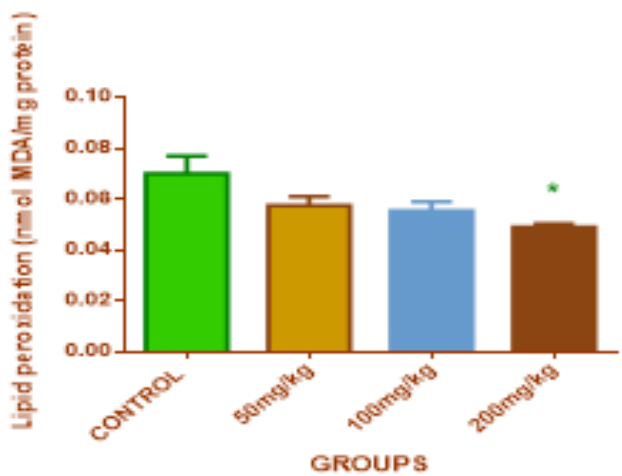

Figure 9: Oral administration of 3PFBPA inhibited mitochondrial lipid peroxidation.

3PFBPA: 3-para-fluorobenzoyl-propionic acid

* Each value is statistically significant at $\mathbf{p}<0.05$, compared with control using the one-way

\subsection{Discussion}

Haloperidol is a classical antipsychotic drug used to treat an array of psychotic disorders. Two major pathways have been identified for haloperidol metabolism. One is a reversible reductive pathway that produces 4-(4-chlor- ophenyl)- $\alpha$-(4-fluorophenyl)-4-hydroxy-1piperidinebutanol while the other is an oxidative $\mathrm{N}$-dealkylation pathway that leads to two products: 4-(4-chlorophenyl)-4hydroxypiperidine and 3-(4-fluorobenzoyl) propionic acid (3PFBPA) (Bowen et al., 1990). The 3PFBPA has been shown to be an inhibitor of MAPK. However its effect on $\mathrm{mPT}$ pore has not yet been investigated, thus; the reason for this study. In the present study, we found that the membrane integrity of the mitochondria used for this study were not compromised, hence, the mitochondria were intact, not uncoupled and suitable for use. When intact mitochondria were exposed to 3PFBPA, the $\mathrm{mPT}$ pore openend significantly compared to NTA. Moreover, the opening of the pore was reversed by spermine showing that 3PFBPA induction of pore opening had no deleterious effect on membrane integrity. These therefore suggest that 3PFBPA could possibly interact with the components of the pore, cause the opening of the pore and ultimately apoptosis. Moreover, the effect was concentrationdependent.

Cytochrome c release due to opening of pore has been shown to be critical for for the induction of mitochondrial-mediated apoptosis (Green and Kroemer, 2004). The results on cytochrome c suggest that there was mPT pore opening caused by 3PFBPA which resulted in cytochrome c release. This is sine qua non for apoptosis.

The status of mitochondrial $\mathrm{F}_{0} \mathrm{~F}_{1}$-ATPase was investigated in the presence of varying concentrations of 3PFBPA using 2,4Dinitrophenol (DNP), as a standard. This was determined using spectrophotometer by following the rate of release of inorganic phosphate. 3PFBPA significantly caused the release of inorganic phosphate in a concentration-dependent manner as measured by their ATPase activity. These results suggest that 3PFBPA could interact with the pore and as a result, caused $\mathrm{MPT}$ pore opening and subsequent cytochrome c release which is a crucial step for mitochondrialmediated apoptosis to occur (Kallenberger et al., 2014). These probably suggest that 3PFBPA may be considered as a potential drug candidate in situations where apoptosis needs to be upregulated, such as cancer. Also, the result is consistent with reports that mPT pore opening causes uncoupling of oxidative phosphorylation by inhibiting uptake of inorganic phosphate. This converts mitochondria to ATP consumers instead of producers, thus, elevating inorganic phosphate level (Bernardi and Di Lisa, 2015). The results from the lipid peroxidation show that varying concentrations of 3PFBPA inhibited lipid peroxidation. Compounds that inhibit membrane phospholipids peroxidation may be relevant in preventing free radical-induced damage (Ruberto et al., 2000).

Conversely, the in vivo study did not show 3PFBPA to be an inducer of $\mathrm{mPT}$ pore opening as there was no effect on mPT pore opening compared with the control. Also, mitochondrial ATPase activity was not enhanced. This could occur by reason of biotransformation converting the 3PFBPA into another metabolite that does not induce 
mPT pore opening since the route of administration is oral. There are several mechanisms of action by which an anti-cancer drug could elicit its pharmacological effect apart from induction of $\mathrm{mPT}$ pore opening (Islam et al., 2014; MdSoriful et al., 2017). Interestingly, the in vivo study (figure 9) showed the anti-lipid peroxidative activity of 3PFBPA on the mitochondrial membrane. This is in consonance with our in vitro results. This also suggests that 3PFBPA may protect the physicochemical properties of biological membrane from lipid peroxidationinduced damage.

In conclusion, based on the findings from this study, it is suggested that 3PFBPA, a metabolite of anti psychotic drug, should be further investigated on its ability to induce apoptosis (either through mitochondrial-mediated or extrinsic pathway), and inhibit mitochondrial lipid peroxidation.

\section{Conflict of Interest}

There is no conflict of interest to declare among the authors.

\section{Authors' Contributions}

Conception: [AOO]

Design: [AOO and 000]

Execution: [AOO and OLB]

Interpretation: [AOO and $\mathrm{OLB}]$

Writing the paper: [AOO]

\section{References}

Appaix, F., Minatchy, M., Riva-Lavieille, C., Olivaires, J., Antonnson, B., Saks, V.A., 2000, Rapid spectrophotometric method for quantitation of cyctochrome c release from isolated mitochondria or permealized cells revisited. Biochimica et Biophysica Acta, 1457: 175-181.

Bassir, O., 1963, Improving the level of nutrition. West African Journal of Biology and Applied Chemistry, 7: 32-40.

Bernardi, P., Di Lisa, F., 2015, The mitochondrial permeability transition pore: Molecular nature and role as a target in cardioprotection. Journal of Molecular and Cellular Cardiology, 78:100-106.

Bowen, W.D., Moses, E.L., Tolentino, P.J., Walker, J.M., 1990, Metabolites of haloperidol display preferential activity at $\sigma$ receptors compared to dopamine D-2 receptors. Eur J Pharmacol, 177: 111-118.

Green, D.R., Kroemer, G., 2004, The pathophysiology of mitochondrial cell death. Science, 305 (5684): 626-629.

Huang, S., Hlzel, M., Knijnenburg, T., 2012, MED12 controls the response to multiple cancer drugs through regulation of TGF-beta receptor signaling. Cell, 151: 937-50.

HyeonSoo Kim, Minseok Song, SanatombiYumkham, Jang Hyun Choi, Taehoon Lee, Joseph Kwon, Sung Jae Lee, Jong-In Kim, Kang Woo Lee, Pyung-Lim Han, Seung Woo Shin, Ja-Hyun Baik, Yong Sik Kim, Sung Ho Ryu and Pann-GhillSuh, 2006, Identification of a new functional target of haloperidol metabolite: implications for a receptor-independent role of 3-(4-fluorobenzoyl) propionic acid. Journal of neurochemistry, 2006: 10-13.

Jiang, Y., Yang, J., Yang, C., 2013, Vitamin K4 induces tumor cytotoxicity in human prostate carcinoma PC-3 cells via the mitochondria-related apoptotic pathway. Pharmazie, 68: 442-448.

Kallenberger S., Joël B., Juliane C., Carmen F., Peter, K.S, Stefan L., 2014, Intra- and Interdimeric Caspase-8 Self-Cleavage Controls Strength and Timing of CD95-Induced Apoptosis. Sci. Signal, 7 (316): 23.

Lee, E., Min, H., Park, H., Chung, H., Kim, S., Han, Y., Lee, S., 2004, G2/M cell cycle arrest and induction of apoptosis by a stilbenoid, 3,4,5trimethoxy-49-bromo-cis-stilbene, in human lung cancer cells. Life Sci, 75: 2829-2839.

Lowry, O.H., Rosenbrough, N.J., Farr, A.L., Randall, R.J., 1951, Protein measurement with Folin phenol reagent. J Biol Chem, 193 (1): 265275.

Martins, K.R., 2006, Targeting apoptosis with dietary bioactive agents. Minireview, 117-130.

Mcllwain, D. R., Berger, T., Mak, T.W., 2013, Caspase functions in cell death and disease. Cold Spring Harbor Perspecives in Biology, 1 (5): 4.

MdSoriful Islam, Most Mauluda Akhtar, James H. Segars, Mario Castellucci, Pasquapina Ciarmela, 2017, Molecular targets of dietary phytochemicals for possible prevention and therapy of uterine fibroids: Focus on fibrosis, Critical Reviews in Food Science and Nutrition, 57 (17): 3583-3600.

Olorunsogo, O.O., Malomo, S.O., 1985, Sensitivity of Oligomycininhibited respiration of isolated rat liver mitochondria to perfluidone, a fluorinated arylalkylsulfonamide. Toxicology, 35(3): 231-40.

Olorunsogo, O.O., Bababunmi, E.A., Bassir, O., 1979, Uncoupling effect of N-phosphonomethylglycine on rat liver mitochondria. Biochem. Pharm, 27: 925-927.

Olowofolahan, O.A., Ezekiel, C.D., Olorunsogo, O.0., 2019, Induction of Mitochondrial Membrane Permeability Transition Pore Opening and DNA Fragmentation by Certain Solvent Fractions of Mangifera indica. Archives of Basic and Applied Medicine, 7: 123 - 129.

Reed, J., 2004, Apoptosis mechanisms: implications for cancer drug discovery. Oncology, 18:11-20.

Ruberto, G., Baratta, M.T., Deans, S.G, Dorman, H.J., 2000, Antioxidant and antimicrobial activity of Foeniculum vulgare and Crithmum maritimum essential oils. Planta Med, 66: 687-693.

Varshney, R., Kale, R.K., 1990, Effect of calmodulin antagonists on radiation-induced lipid peroxidation in microsomes. Int. Radiat Biol, 58: 773-743. 